EPJ Web of Conferences 37, 01013 (2012)

DOI: $10.1051 /$ epjconf/20123701013

(C) Owned by the authors, published by EDP Sciences, 2012

\title{
First pionic atom spectroscopy at RIBF
}

Kenta Itahashi ${ }^{1, \mathrm{a}}$, Georg P.A. Berg ${ }^{2}$, Hiroyuki Fujioka ${ }^{3}$, Hans Geissel ${ }^{4}$, Ryugo S. Hayano ${ }^{5}$, Satoru Hirenzaki ${ }^{6}$, Natsumi Ikeno ${ }^{6}$, Naohito Inabe ${ }^{1}$, Satoshi Itoh $^{5}$, Daisuke Kameda ${ }^{1}$, Toshiyuki Kubo $^{1}$, Hiroaki Matsubara ${ }^{7}$, Shinichiro Michimasa ${ }^{7}$, Kenjiro Miki ${ }^{8}$, Hiroyuki Miya ${ }^{7}$, Masaki Nakamura ${ }^{1}$, Takahiro Nishi ${ }^{5}$, Shumpei Noji ${ }^{7}$, Shinsuke Ota ${ }^{7}$, Ken Suzuki $^{9}$, Hiroyuki Takeda ${ }^{1}$, Koichi Todoroki ${ }^{5}$, Kyo Tsukada ${ }^{10}$, Tomohiro Uesaka ${ }^{1}$, Helmut Weick ${ }^{4}$, and Koichi Yoshida ${ }^{1}$

1 RIKEN Nishina Center, RIKEN, 351-0198 Saitama, Japan

2 JINA and Department of Physics, University of Notre Dame, Indiana 46556, USA

3 Division of Physics and Astronomy, Kyoto University, 606-8502 Kyoto, Japan

4 GSI - Helmholtzzentrum für Schwerionenforschung GmbH, D-64291 Darmstadt, Germany

5 Department of Physics, University of Tokyo, 113-0033 Tokyo, Japan

Department of Physics, Nara Women's University, 630-8506 Nara, Japan

Center for Nuclear Study, University of Tokyo, 351-0198 Saitama, Japan

RCNP, Osaka University, 567-0047 Osaka, Japan

9 Stefan-Meyer-Institut für subatomare Physik Austrian Academy of Sciences, Austria

10 Department of Physics, Tohoku University, 980-8578 Miyagi, Japan

\begin{abstract}
We have performed an inclusive spectroscopy of ${ }^{122} \mathrm{Sn}\left(d,{ }^{3} \mathrm{He}\right)$ reaction near the pion emission threshold at an incident energy of $T_{d}=250 \mathrm{MeV} /$ nucleon. The first experiment aims as devloping the high precision spectroscopy of pionic atoms at the RI beam factory (RIBF) of RIKEN, which leads to a new project, pionic Atom Factory project (piAF) to measure pionic atoms for a wide range of elements. We report the analysis status of the pilot experiment.
\end{abstract}

\section{Introduction}

In order to understand the non-perturbative aspects of the QCD in the low energy region, it is important to understand the chiral SU(3) dynamics where hadrons behave as the degree of freedom. The chiral symmetry is spontaneously broken and is parametrized by chiral condensate $\langle\bar{q} q\rangle$, which varies as a function of the temperature and the density of the medium [1,2]. Among experimental approaches to study chiral symmetry and its spontaneous breaking, in-medium spectroscopy of hadrons has been playing an important role. One of the preceding works is a high precision spectroscopy of deeply bound pionic atoms in $\left(d,{ }^{3} \mathrm{He}\right)$ reactions at GSI [3-6]. The $\langle\bar{q} q\rangle$ condensate at the normal nuclear density is found to be $\sim 30 \%$ smaller than in vacuum [6-8]. Although the evaluation is still associated with large statistical and systematic errors, precision spectroscopy of in-medium hadrons proved itself capable of setting constraints on the dynamically-breaking chiral symmetry.

Now, we performed an experiment to measure pionic atoms at RIBF for the first time. The employed reaction was ${ }^{122} \mathrm{Sn}\left(d,{ }^{3} \mathrm{He}\right)$ to produce pionic ${ }^{121} \mathrm{Sn}$ atoms. According to theoretical works, we expect observation of $(1 s)_{\pi}$ state as a distinct peak in the excitation spectrum.

We will report here the present status of the spectral analysis and future perspectives.

a e-mail: itahashi@riken.jp

This is an Open Access article distributed under the terms of the Creative Commons Attribution License 2.0, which permits unrestricted use, distribution, and reproduction in any medium, provided the original work is properly cited. 


\section{Experiment}

Comparing facilities RIBF and GSI from the viewpoint of pionic atom spectroscopy, the largest advantage of RIBF is its high intensity primary beam. RIBF has a DC continuous beam with an intensity of $10^{12} / \mathrm{s}$ while GSI provides $10^{11}$ /spill with the duty factor of $16 \%$. Thus, effectively, we have more than 10 times higher intensity in RIBF, which allows adoption of thinner target leading to better resolution potentially. The largest problem of the RIBF is the intrinsic beam momentum spread which is evaluated to be $\sim 0.1 \%$ and the corresponding resolution was estimated to be $\sim 1 \mathrm{MeV}$ (FWHM). Comparing to GSI, the resolution is more than twice worse.

In order to achieve better resolution eliminating the contribution from the beam momentum spread, one way is to reduce the beam momentum spread using slits at a dispersive focal plane. This will result in activation of the facility and will reduce the beam intensity. We have developed another way by constructing a special beam optics. The incident beam momentum spread is analyzed on the target with a dispersion matching condition to a central focal plane, and the contribution from the momentum spread is eliminated from the focal plane spectra.

\subsection{Pilot Experiment}

In order to demonstrate the feasibility and establish the necessary experimental conditions, we performed a pilot experiment. This R\&D effort involves many aspects including beam track calculations in the accelerators, beam optics, detectors, and electronics. The pilot experiment was carried out in October 2010 for the data taking period of three days aiming at (i) test of the detector performance under extremely high rate conditions of $\sim 30 \mathrm{MHz}$, (ii) investigation of the spectral resolution, and (iii) overall test of the conditions of systematic studies of pionic atoms. Our goal was the world highest spectral resolution of $\sim 400 \mathrm{keV}$ (FWHM) with a high intensity beam of $\sim 10^{12}$ deuterons/sec.

Figure 1 depicts a schematic view of the RIBF facility [9]. The deuteron beam is accelerated by the SRC cyclotron to $T_{d}=250 \mathrm{MeV} /$ nucleon with the maximum intensity of $\sim 10^{12} / \mathrm{s}$. We placed a ${ }^{122} \mathrm{Sn}$ target with the thickness of $10 \mathrm{mg} / \mathrm{cm}^{2}$ at the target position.

${ }^{3} \mathrm{He}$ particles emitted in the $\left(d,{ }^{3} \mathrm{He}\right)$ reaction are momentum-analyzed by the first part of the BigRIPS beamline used as a magnetic spectrometer. The central focal plane (F5) is set to be a dispersive focal plane with the dispersion of about $6 \mathrm{~m}$. A set of two multi-wire drift chambers are installed at F5 to measure the ${ }^{3} \mathrm{He}$ tracks. The second part is used mainly for particle identification purposes. We installed two sets of scintillation counters at F5 and F7 to measure the energy loss of particles and the time of flight (TOF) between the F5 and F7 focal planes. The instantaneous particle rate at the F5 scintillation counters amounted to $\sim 35 \mathrm{MHz}$ mainly due to the breakup reaction of the deuteron beam in the target.

The trigger for the data acquisition system is provided by the scintillation counters. We have adjusted the coincidence between F5 and F7 scintillation counters to apply rough selection of particles based on the TOF. The coincidence trigger rate was suppressed to less than $100 \mathrm{~Hz}$.

Figure 2 shows the particle identification performance of the detector system. The abscissa is the measured energy loss in the scintillation counter at F5 and the ordinate is the TOF measured between the F5 and F7 scintillation counters. As clearly seen, ${ }^{3} \mathrm{He}$ is identified without any contamination.

\subsection{Experimental Results}

Figure 3 shows the measured acceptance-corrected spectrum of the $\left(d,{ }^{3} \mathrm{He}\right)$ reaction at $T_{d}=250$ $\mathrm{MeV} /$ nucleon. The spectrum reprents the data accumulated in a 16-hour measurement. We still need to finalize the calibrations of the the ${ }^{3} \mathrm{He}$ energy and the cross section of the reaction. Thus, both abscissa and the ordinate are still preliminary and will be adjusted once the analysis is finalized.

The overall structure of the spectrum is understood by comparison to the previous experiments and to the theoretical predictions. We see a continuum ranging from left side below about $355 \mathrm{MeV}$ ${ }^{3} \mathrm{He}$ kinetic energy which is attributed to quasi-free pion production. The three peaks observed in the 


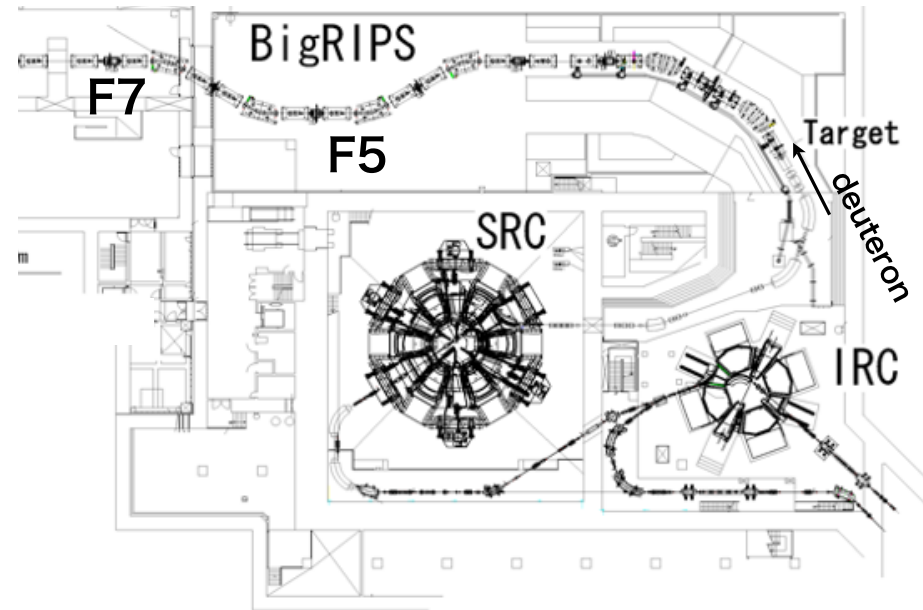

Fig. 1. A schematic view of the experimental site, RIBF [9]. The $T_{d}=250 \mathrm{MeV} /$ nucleon deuteron beam is accelerated by the cyclotron SRC and impinges on the target. The ${ }^{3} \mathrm{He}$ particles are momentum analyzed and measured at the dispersive focal plane F5. The F5-F7 section is used for particle identification purposes.

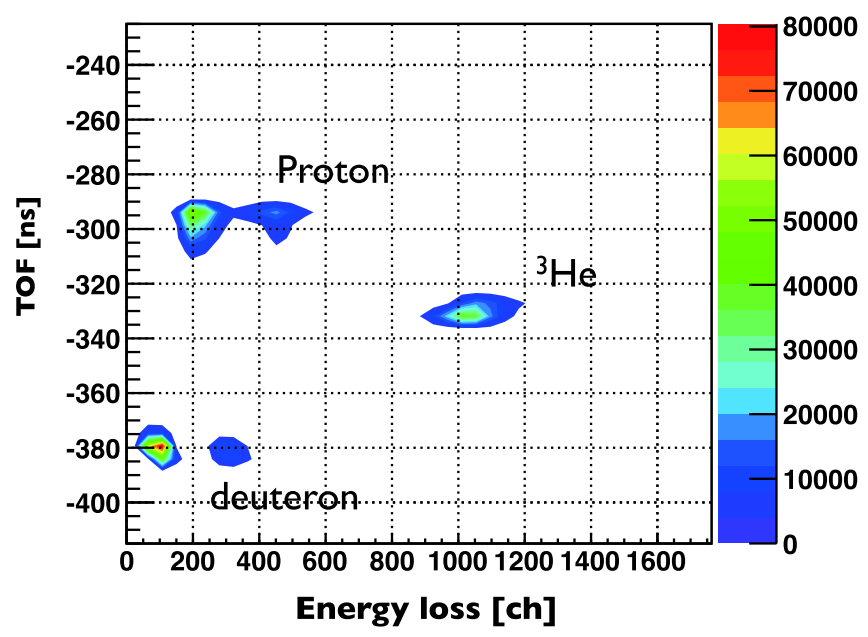

Fig. 2. Particle identification capability of the detector system by measuring the TOF between F5 and F7 and the energy loss in the F5 scintillator. ${ }^{3} \mathrm{He}$ particles are identified with perfect background rejection.

energy region between 357 and $362 \mathrm{MeV}$ are due to the first experimental observation of pionic ${ }^{121} \mathrm{Sn}$ atoms. The properties of the three peaks are being discussed.

Figure 4 depicts the reaction angle at the target vs. the roughly calibrated energy. This is the first observation of the angular dependence of the pionic atom formation cross section in the $\left(d,{ }^{3} \mathrm{He}\right)$ reaction.

Figure 5 shows the measured roughly acceptance-corrected position spectrum with the condition of $<15 \mathrm{mrad}$ for the reaction angle. The peaks to the left are smaller compared to the right-most peak. Figure 6 shows the theoretically calculated $Q$-value spectrum of the $\left(d,{ }^{3} \mathrm{He}\right)$ reaction assuming an experimental resolution of $150 \mathrm{keV}$ (FWHM) [10] for comparison. Note that the quasi-free component 


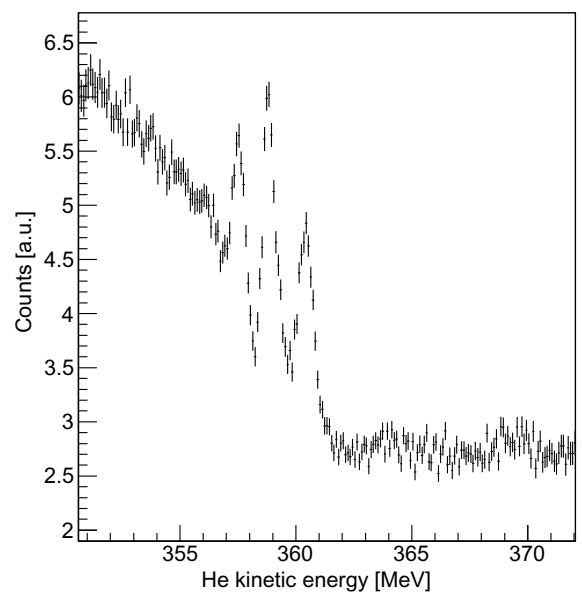

Fig. 3. Measured roughly acceptance-corrected spectrum of the $\left(d,{ }^{3} \mathrm{He}\right)$ reaction near the pion-emission threshold. The calibrations of the abscissa is preliminary.

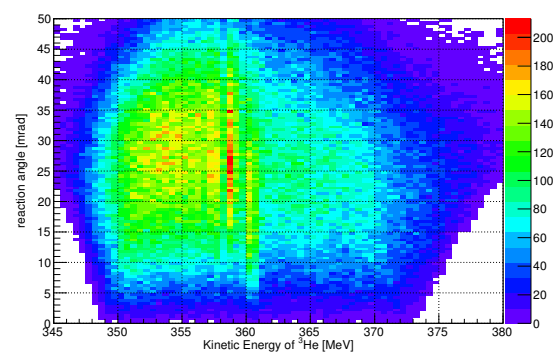

Fig. 4. Measured reaction angle as function of the kinetics energy.

is not included in the calculation. Overall structures in the figures agree well, and the most prominent peak is assigned to a configuration of $(1 s)_{\pi} \otimes\left(3 s_{1 / 2}\right)_{n^{-}}$.

\section{Conclusion and Future Perspectives}

We have successfully observed for the first time a spectrum of ${ }^{122} \mathrm{Sn}\left(d,{ }^{3} \mathrm{He}\right)$ reaction near the $\pi^{-}$ emission threshold at an incident deuteron energy of $250 \mathrm{MeV} /$ nucleon. The spectrum was measured at RIBF. The BigRIPS beamline was used as a forward angle spectrometer. The large acceptance of BigRIPS allowed the first measurement of the angular dependence of the pionic-atom formation cross section by using the $\left(d,{ }^{3} \mathrm{He}\right)$ reaction.

The overall experimental resolution is estimated to be similar to the value of $400 \mathrm{keV}$ (FWHM) previously achieved at GSI. A simple estimation of the resolution would exceed $1 \mathrm{MeV}$ due to the incident beam momentum spread of $0.1 \%$ which is a factor of three worse than GSI. The achievement of $400 \mathrm{keV}$ is due to development and application of a dispersion matching beam optics.

We observed ${ }^{121} \mathrm{Sn}-1 s$ pionic atom as a distinct large peak and the $2 s$ also as a small peak. We are presently working on higher-order aberration corrections of the beam optics, acceptance correction, and calibration of the spectra. The information on the binding energies and widths will be deduced in the extensive analysis that follows.

Note that the spectrum presented in this report was measured in a very short time of 16 hours. The experiment revealed the potential capability of the RIBF facility as a pionic-atom factory to cover 


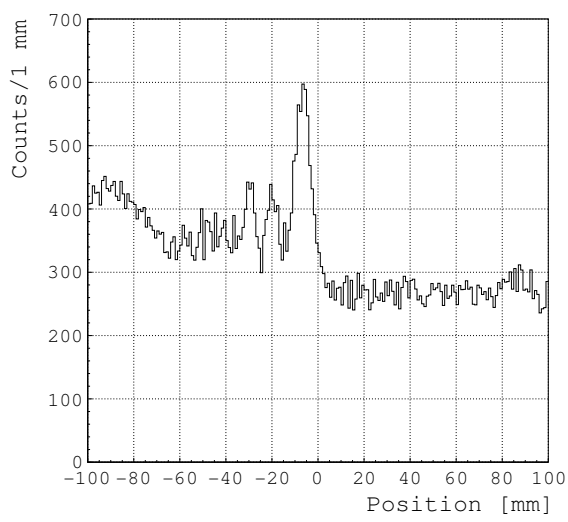

Fig. 5. Measured acceptance-corrected position spectrum with a condition of the reaction angle $<15$ mrad.

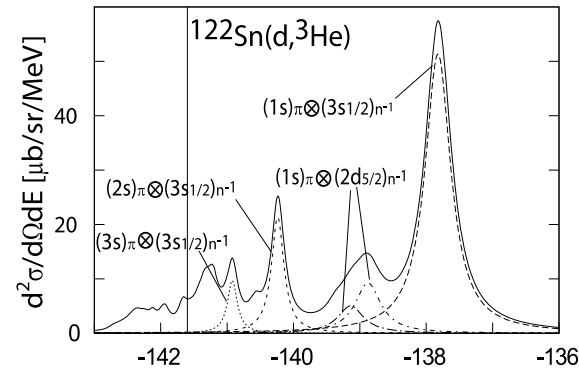

Fig. 6. Theoretical calculation by Ikeno et al. with an assumed resolution of $150 \mathrm{keV}$ (FWHM) [10]. The quasifree component is not included in the calculation. Comparison of the figures of the calculation and the data of Fig. 5 lead to the assignment of the largest peak to a configuration of $(1 s)_{\pi} \otimes\left(3 s_{1 / 2}\right)_{n^{-}}$.

a wide range of elements. We are going to optimize the experimental procedure in order to achieve higher resolution with smaller systematic errors in the near future. This work is partly supported by the Grants-in-Aid for Scientific Research (22105517, 20540273, 22105510, 24105712), JSPS fellows (No. 23·2274), and Specially Promoted Research (20002003).

\section{References}

1. U. Vogl and W. Weise, Prog. Part. Nucl. Phys. 27, 195 (1991) and references therein.

2. T. Hatsuda and T. Kunihiro, Phys. Rept. 247, 221 (1994) and references therein.

3. H. Gilg et al., Phys. Rev. C62, 025201 (2000).

4. K. Itahashi et al., Phys. Rev. C62, 025202 (2000).

5. H. Geissel et al., Phys. Rev. Lett. 88, 122301 (2002).

6. K. Suzuki et al., Phys. Rev. Lett. 92, 072302 (2004).

7. E. E. Kolomeitsev, N. Kaiser, and W. Weise, Phys. Rev. Lett. 90, 092501 (2003).

8. T. Hatsuda and T. Kunihiro, Prog. Theor. Phys. 74, 765 (1985).

9. T. Kubo et al., Nucl. Instr. Meth. in Phys. Res. B, 204, 97(2003).

10. N. Ikeno et al., Prog. Theor. Phys., 126, 483 (2011). 